\title{
Ambulatory pulmonary arterial pressure in primary pulmonary hypertension: variability, relation to systemic arterial pressure, and plasma catecholamines
}

\author{
A Mark Richards, Hamid Ikram, Ian G Crozier, M Gary Nicholls, Siegfried Jans
}

\begin{abstract}
The variability of pulmonary arterial pressure, the relation of pulmonary pressure to systemic pressure, pulmonary pressure responses to stimuli (exercise, hypoxia, smoking, free ambulation), and plasma catecholamine responses were assessed in five patients with primary pulmonary hypertension. Ambulatory monitoring techniques provided data for the computerised analysis of continuous, beat-to-beat, direct recordings of both pulmonary and systemic arterial pressures for 8 to 10 hours. The absolute variability of pulmonary arterial pressure and the magnitude of absolute changes in this variable in response to stimuli were increased in primary pulmonary hypertension. The variability of systemic pressure was similar to that in healthy volunteers. Basal and stimulated plasma catecholamine values were normal, suggesting preservation of normal sympathetic nervous system activity in primary pulmonary hypertension.
\end{abstract}

Primary pulmonary hypertension is a rare disease $^{1}$ and its cardinal characteristic, an increase in pulmonary arterial pressure, is difficult to measure without catheterisation of the pulmonary artery. For these reasons data on levels and variability of pulmonary artery pressure in primary pulmonary hypertension are scant and confined to intermittent readings in restricted, catheterised patients. ${ }^{23}$ Spontaneous variability in pulmonary pressure has been put forward as a major impediment to the assessment of responses in therapeutic trials of vasodilator agents in primary pulmonary hypertension. However, our knowledge of the magnitude of such variation is very limited. ${ }^{23}$ Further, in these patients, the behaviour of the pulmonary circulation during unrestricted ambulation and how responses to physiological stimuli may differ from normal are quite unknown. It is also not known whether the relation between concomitant shifts in systemic and pulmonary arterial pressures is distorted in primary pulmonary hypertension. Finally, the activity of the sympathetic nervous system in primary pulmonary hypertension is largely unexplored. We have previously reported a tech- nique which allows the continuous recording of pulmonary artery pressure in unrestricted people over prolonged periods ${ }^{4}$ and described the range and variability of pulmonary artery pressure and its relations to systemic arterial pressure and plasma catecholamines in healthy volunteers during several everyday activities. ${ }^{5}$ The current report is the first to describe alterations in these variables at rest and in response to exercise, eating, smoking, and hypoxia in ambulant patients with primary pulmonary hypertension and it compares these findings with those reported in earlier studies of healthy volunteers.

\section{Patients and methods}

The protocol was approved by the hospital ethics committee and all patients gave informed consent. The study group consisted of five patients (four women and one man, aged 24 to 60 years (mean 41 years)) with primary pulmonary hypertension (mean pulmonary artery pressure $>25 \mathrm{~mm} \mathrm{Hg}$ at rest, no secondary cause identified by full evaluation ${ }^{6}$ ). We compared the results with those in six healthy people (three women, three men) aged 24 to 40 years (mean 29 years) who were studied earlier. ${ }^{5}$ Three patients were treated with captopril (25 mg twice or three times a day) and two were untreated.

The patients were studied on day 4 of a diet containing a constant amount of sodium $(80 \mathrm{mmol} /$ day) and potassium $(60 \mathrm{mmol} /$ day $)$ and no caffeine. Between 0800 and 1000 hours on the study day they underwent pulmonary artery catheterisation via an antecubital vein with a fluid filled F5 Goodale-Lubin catheter. The catheter was connected to a portable transducer-perfusion unit as already described. ${ }^{4}$ After careful clinical assessment of arterial blood supply to the left arm, the left brachial artery was cannulated with a F3 Seldicath connected to a second portable transducer-perfusion unit. The left radial pulse and circulation in the left hand were checked throughout the study. Signals from the two transducers, together with the electrocardiogram from chest leads, were continuously recorded on separate channels of a Medilog miniaturised, multichannel tape recorder worn on a belt at the waist. The pulmonary arterial pressure signal was processed by an AM4 recorder module set for linear amplification of pressures between 0 and $150 \mathrm{~mm} \mathrm{Hg}$. The 
frequency response for both systemic and pulmonary artery pressure was linear to $8 \mathrm{HZ}$. The methods of continuous ambulatory recording of both pulmonary and systemic arterial pressures have been described elsewhere. ${ }^{457}$ To eliminate zero shift problems caused by postural changes with the low pressure pulmonary system the transducers were worn on the anterior chest wall directly over the tip of the catheter in the main pulmonary artery (placed under flucroscopic guidance) and the patients remained upright, either seated or standing, throughout the recording period. Both transducers were calibrated with a mercury column at the beginning and at the end of each study and also at approximately the midpoint of the protocol.

After catheterisation, patients were given breakfast and allowed a period of 1.5 hours to become accustomed to their portable equipment. Each participant then progressed through a sequence of interventions. These were spaced at least an hour apart, and between interventions patients were able to move about, talk, sit, read, sleep, stand, and visit the toilet while recordings continued. Careful note of body posture and activity was made throughout by one observer who used a stopwatch and diary. As a safety measure, an additional member of the research medical staff was present at all times.

Exercise was performed on a stationary upright bicycle with a graded submaximal exercise test (modified Naughton protocol ${ }^{8}$ ) to $75 \%$ of maximum predicted heart rate. Blood was sampled for plasma catecholamine concentrations 10 minutes before exercise $(-10 \mathrm{~min}$ utes) and when exercise started ( 0 minutes) and at the peak exercise as well as $15,20,30$, and 60 minutes after the end of exercise.

Hypoxia-The patients breathed a gas mixture of $10 \%$ oxygen in nitrogen for 30 minutes, or as long as it was tolerated, while in the sitting position. During this intervention, arterial samples for blood gas analysis together with venous blood for catecholamines were drawn at -10 minutes, 0 minutes, peak hypoxia, and 30 minutes after return to breathing room air.

Smoking-Three patients (smokers) smoked two commercial filter tipped cigarettes $(5.5 \mathrm{~cm}$ of each) in 10 minutes. Venous plasma catecholamine samples were taken at 0 and 10 minutes.

Eating-Patients ingested a $2.5 \mathrm{MJ}$ (man) or $2.0 \mathrm{MJ}$ (women) lunch over a period of 15 minutes.

Venous samples were collected into chilled tubes, centrifuged at $4^{\circ} \mathrm{C}$, and stored at $-80^{\circ} \mathrm{C}$ before radioenzymatic assay for plasma catecholamines. ${ }^{9}$

For data analysis, a PDP-11 computer was programmed to calculate average systolic, mean, and diastolic pulmonary and systemic pressures and heart rate for successive groups of 10 heartbearts. These results were used to provide graphical and digital printouts with means and standard deviations for periods of interest. Mean values of pressures and heart rate were calculated for each of the 16-20 half hour periods of total recording time. The short term variability of these variables was assessed by the method of Mancia et $a l^{10}$ in which the standard deviations and coefficients of variation were calculated for each separate half hour period. An index of longer term variability was provided by the standard deviation and variation coefficient derived from averaging the 1620 mean half hourly values. Two way analysis of variance with repeated measures and the $t$ test for paired data were used to test for significant changes in pressure and heart rate and hormone concentrations during interventions. Successive 1 minute mean values of pulmonary and systemic pressures were correlated (product-moment correlation coefficient) during the periods of interest. To compare resting and intervention related plasma concentrations of catecholamines, systemic and pulmonary arterial pressures, heart rate, and the variability of the haemodynamic indices in patients and healthy volunteers studied earlier we used the non-parametric Mann-Whitney test. ${ }^{11}$ Intergroup comparisons were also made by analysis of variance (BMDP program P2V) with "group" (normal or pulmonary hypertensive) and "time" as repeated measures. Interactions between "group" and "time" were inspected to allow detection of significant non-parallelism between groups in the response of pressures to any given stimulus. Data are given as mean (SEM) unless stated otherwise.

\section{Results}

Studies were completed without complications. On day 3 of the constant diet 24 hour urinary excretion of sodium was 69 (5) mmol.

For the 8-10 hour period of continuous recording, mean values for pulmonary and systemic pressures $(\mathrm{n}=5)$ were $49 \cdot 2(8 \cdot 2) / 23 \cdot 0$ (4.8) and $115 \cdot 4(4 \cdot 7) / 65 \cdot 2(24) \mathrm{mm} \mathrm{Hg}$ respectively. Pulmonary, but not systemic, pressures were significantly greater than the corresponding values in our earlier study of healthy volunteers $(15.7 / 6.3 \mathrm{~mm} \mathrm{Hg}(\mathrm{p}<0.01)$ and $121 \cdot 1 / 72 \cdot 4 \mathrm{~mm} \mathrm{Hg}$ (NS) respectively (fig 1). Pulmonary pressure showed similar variability within half hour periods as between half hour periods and in both cases absolute variations ( $\mathrm{mm} \mathrm{Hg}$ ) but not percentage coefficients of variation significantly exceeded values in healthy volunteers (table 1). Over 8-10 hours the variation in systolic pulmonary arterial pressure in individual patients at rest (that is seated, unstimulated, and a minimum of 5 minutes clear of any activity or intervention) was $20-32 \mathrm{mmHg}$ (mean $23 \cdot 2(2 \cdot 2) \mathrm{mm} \mathrm{Hg}$ ). Pulmonary diastolic pressures at rest varied by 8-15 mm Hg (mean 12.2 (1.9) $\mathrm{mm} \mathrm{Hg}$ ) Corresponding values for the mean range covered by resting pulmonary pressures in our volunteer group were significantly lower $(12 \cdot 1$ (1.2) and $6.8(1.3) \mathrm{mm} \mathrm{Hg}$ for systolic and diastolic pressures respectively) ( $p<0.01$ for both comparisons). Swings in resting pulmonary pressure lasted several minutes and occurred gradually, and no precipitous changes (that is occurring within seconds) were recorded at rest. Changes in resting pulmonary pressure bore no clear relation to time of 


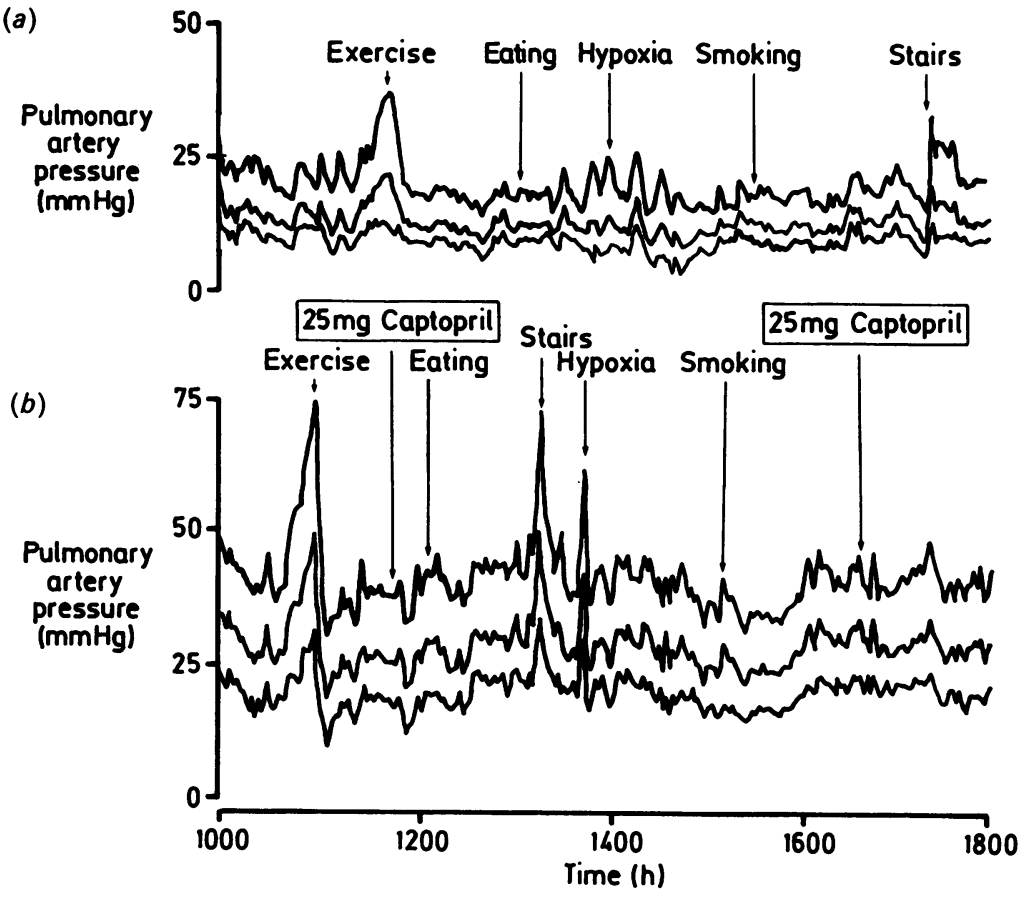

Figure 1 Continuous recordings of pulmonary systolic, mean, and diastolic pressures plotted from serial integrated one minute mean values over 8 hours and during several activities and interventions. (a) Data from a healthy volunteer and (b) a patient with primary pulmonary hypertension (both aged 28 years).

captopril dose (and were similar in both treated and untreated patients) or any other easily discernible external stimulus. Coefficients of variation (SD mean \%) for entire recordings in these patients for pulmonary systolic and diastolic pressures were $15 \cdot 1$ and $20.9 \%$ respectively, within half hours (table 1), an index of short term fluctuation. Coefficients from data entirely confined to recordings taken in seated, resting patients (at least 100 minutes of recording per patient derived from integrated one minute mean values) were $10.6 \%$ and $15.2 \%$ for pulmonary systolic and diastolic values respectively.

The variability of both systemic arterial pressure and heart rate did not significantly differ from normal (table 1). Table 2 gives pressures and heart rates averaged for the total recording period and for separate interventions.

Inspection of computer graph printouts generally showed that pulmonary pressure, sys- temic pressure, and heart rate moved in parallel (figs 2 and 4)-except during hypoxia when pulmonary pressure clearly rose in the absence of a significant change in systemic pressure (fig 3). Overall, for serial 30 minute integrated values, there was a positive correlation between mean pulmonary and systemic pressures in three patients ( $\mathrm{r}$ values $0.58,0.68,0.72$; $\mathrm{p}<0.01$ to $\mathrm{p}<0.001$ ), but this correlation was not found in two patients $(r=0.12,0.22$ (NS)). This variable relation between systemic and pulmonary pressures contrasted with findings in healthy volunteers for whom uniformly strong, positive correlations were found.

Visual comparison of graphic outputs of pulmonary pressures for the entire period of recording from healthy volunteers and patients with primary pulmonary hypertension indicated that the direction of response of pulmonary pressures to any given stimulus was similar in both groups, but the group with pulmonary hypertension showed a more abrupt and greater absolute change (from a higher baseline value) (fig 1). This impression was confirmed by detailed analysis of pressure responses during standardised interventions.

\section{EXERCISE}

The duration of exercise required for patients to achieve $75 \%$ of predicted maximum heart rate varied from 13 to 20 minutes (mean 16.6 $(2 \cdot 7)$ ) and tended to be shorter than in healthy volunteers $(21 \cdot 2(3 \cdot 3)$ minutes, difference NS). Pressures in both pulmonary and systemic circuits increased abruptly at first and then continued a more gradual climb until exercise was halted $(p<0.001$ for changes from resting values in both pulmonary and systemic pressures and systolic and diastolic pressures (fig 2). Throughout exercise pulmonary, but not systemic, pressures were significantly greater than in the healthy volunteers $(p<0.001$, $\mathrm{p}<0.01$ for systolic and diastolic values respectively, table 2) and group-time interaction analysis showed a significantly steeper rise in pulmonary pressures but not systemic pressures with time in the patient group $(p<0.001$ for both pulmonary systolic and diastolic pressures). Pulmonary systolic pressures rose by $43 \mathrm{~mm} \mathrm{Hg}$ from rest to peak exercise compared with only $19 \mathrm{~mm} \mathrm{Hg}$ in healthy volunteers. Pulse pressures increased in both circuits in

Table 1 Pulmonary and systemic arterial pressures for recording over 8-10 hours

\begin{tabular}{|c|c|c|c|c|c|c|}
\hline & & \multirow[b]{3}{*}{$\begin{array}{l}\text { Mean (SEM) } \\
(\mathrm{mm} \mathrm{Hg)}\end{array}$} & \multicolumn{4}{|c|}{ Variability } \\
\hline & & & \multicolumn{2}{|c|}{ Within 0.5 h periods } & \multicolumn{2}{|c|}{ Between $0.5 \mathrm{~h}$ periods } \\
\hline & & & $\begin{array}{l}S D \\
(m m H g)\end{array}$ & $\begin{array}{l}C V \\
(\%)\end{array}$ & $\begin{array}{l}S D \\
(m m H g)\end{array}$ & $\begin{array}{l}C V \\
(\%)\end{array}$ \\
\hline \multicolumn{7}{|l|}{ PAP } \\
\hline Systolic & 1 PHT & $49 \cdot 2(8 \cdot 2)^{\star \star}$ & $7 \cdot 2^{\star \star}$ & $15 \cdot 1$ & $7 \cdot 5^{\star}$ & $15 \cdot 3$ \\
\hline \multirow{3}{*}{ Diastolic } & Normal & $15 \cdot 7(1 \cdot 0)$ & 3.8 & $25 \cdot 8$ & 3.5 & $22 \cdot 0$ \\
\hline & $1^{\circ} \mathrm{PHT}$ & $23 \cdot 0(4 \cdot 8)^{\star}$ & $3 \cdot 7^{\star \star}$ & $20 \cdot 9$ & $3 \cdot 8^{\star}$ & $19 \cdot 5$ \\
\hline & Normal & $6.3(0.7)$ & $2 \cdot 4$ & $42 \cdot 7$ & & $31 \cdot 1$ \\
\hline $\begin{array}{l}\text { SAP } \\
\text { Systolic }\end{array}$ & $\begin{array}{l}1 \text { PHT } \\
\text { Normal }\end{array}$ & $\begin{array}{l}115.4(4 \cdot 7) \\
121 \cdot 1(5 \cdot 3)\end{array}$ & $\begin{array}{l}9 \cdot 3 \\
8 \cdot 1\end{array}$ & $\begin{array}{l}8 \cdot 1 \\
6.8\end{array}$ & $\begin{array}{l}8.5 \\
9.3\end{array}$ & $\begin{array}{l}7 \cdot 4 \\
8 \cdot 0\end{array}$ \\
\hline \multirow{2}{*}{ Diastolic } & $1^{\circ} \mathrm{PHT}$ & $65 \cdot 2(2 \cdot 4)$ & $7 \cdot 0$ & $10 \cdot 8$ & $4 \cdot 4$ & $6 \cdot 8$ \\
\hline & Normal & $72.4(3.8)$ & $6 \cdot 0$ & $8 \cdot 7$ & $6 \cdot 0$ & $8 \cdot 3$ \\
\hline \multirow{2}{*}{$\begin{array}{l}\text { Heart rate } \\
\text { (bpm) }\end{array}$} & $1^{\prime} \mathrm{PHT}$ & $89.8(6.3)$ & $6 \cdot 0$ & $6 \cdot 4$ & $6 \cdot 8$ & $6 \cdot 8$ \\
\hline & Normal & $91.0(2.9)$ & $9 \cdot 3$ & $10 \cdot 1$ & $11 \cdot 3$ & $11 \cdot 3$ \\
\hline
\end{tabular}

1 PHT, primary pulmonary hypertension; PAP, pulmonary artery pressure; SAP, systemic arterial pressure; CV, coefficient of variation $\left(\mathrm{SD} / \mathrm{mean}^{\circ}{ }_{0}\right)-\mathrm{n}+5$ for $1^{\prime} \mathrm{PHT}$ and $\mathrm{n}+6$ for healthy volunteers. Significance of difference compared with normal: ${ }^{\star} p<0.05,{ }^{\star} \mathrm{p}<0.01$. 
Table 2 Pulmonary and systemic arterial pressures and heart rate during total recording and interventions (mean (SEM))

\begin{tabular}{|c|c|c|c|c|c|}
\hline & \multicolumn{2}{|c|}{ Pulmonary arterial pressure } & \multicolumn{2}{|c|}{ Systemic arterial pressure } & \multirow[b]{2}{*}{ Heart rate (beats/min) } \\
\hline & Systolic ( $\mathrm{mm} \mathrm{Hg}$ ) & Diastolic ( $\mathrm{mm} \mathrm{Hg}$ ) & Systolic ( $\mathrm{mm} \mathrm{Hg}$ ) & Diastolic ( $\mathrm{mm} \mathrm{Hg}$ ) & \\
\hline $\begin{array}{l}\text { Total recording: } \\
1^{\circ} \mathrm{PHT}(\mathrm{n}=5) \\
\text { Normal }(\mathrm{n}=6)\end{array}$ & $\begin{array}{l}49.2(8.3) \\
15.7(1.0)\end{array}$ & $\begin{array}{r}23.2(4 \cdot 4) \\
6.3(0 \cdot 7)\end{array}$ & $\begin{array}{ll}116 \cdot 1 & (4 \cdot 8) \\
121 \cdot 1 & (5 \cdot 3)\end{array}$ & $\begin{array}{l}65 \cdot 3(2 \cdot 7) \\
72 \cdot 4(3 \cdot 8)\end{array}$ & $\begin{array}{ll}92.3 & (4.5) \\
91.0 & (2.9)\end{array}$ \\
\hline $\begin{array}{ll}\text { Resting: } & \\
1^{\circ} \mathrm{PHT} & (\mathrm{n}=5) \\
\text { Normal } & (\mathrm{n}=6)\end{array}$ & $\begin{array}{l}47 \cdot 1 \quad(6 \cdot 7) \\
13 \cdot 9(1 \cdot 1)\end{array}$ & $\begin{array}{r}22 \cdot 2(4 \cdot 2) \\
5.5(0.9)\end{array}$ & $\begin{array}{ll}114.6 & (6.9) \\
118.7 & (4.9)\end{array}$ & $\begin{array}{l}63 \cdot 4(3 \cdot 2) \\
69 \cdot 0(2 \cdot 7)\end{array}$ & $\begin{array}{ll}91 \cdot 4 & (4 \cdot 1) \\
84 \cdot 8 & (3 \cdot 4)\end{array}$ \\
\hline $\begin{array}{ll}\text { Exercise: } & \\
1^{\circ} \mathrm{PHT} & (\mathrm{n}=5) \\
\text { Normal } & (\mathrm{n}=6)\end{array}$ & $\begin{array}{l}89 \cdot 6(14 \cdot 3)^{\star \star \star} \\
33 \cdot 3 \cdot(1 \cdot 7)\end{array}$ & $\begin{array}{l}37 \cdot 2(6 \cdot 9)^{\star \star \star} \\
12 \cdot 0(0 \cdot 9)^{\star \star \star}\end{array}$ & $\begin{array}{l}166 \cdot 2(14 \cdot 3) \\
157 \cdot 0(7 \cdot 3)\end{array}$ & $\begin{array}{l}80 \cdot 8(4 \cdot 6) \\
85 \cdot 8(4 \cdot 2)\end{array}$ & $\begin{array}{ll}125.4 & (9.4) \\
152.0 & (5 \cdot 4)^{\star \star \star}\end{array}$ \\
\hline $\begin{array}{ll}\text { Eating: } & \\
1^{\circ} \text { PHT } & (n=5) \\
\text { Normal } & (n=6)\end{array}$ & $\begin{array}{ll}53 \cdot 8 & (8 \cdot 1) \\
16.0 & (1.9)\end{array}$ & $\begin{array}{r}25 \cdot 4(4 \cdot 5) \\
7 \cdot 2(1 \cdot 6)\end{array}$ & $\begin{array}{ll}124 \cdot 0 & (8 \cdot 2) \\
129 \cdot 8 & (6 \cdot 7)^{\star}\end{array}$ & $\begin{array}{l}67.6(5.1) \\
79.0(3.7)^{\star}\end{array}$ & $\begin{array}{ll}94 \cdot 2 & (7 \cdot 5) \\
95 \cdot 5 & (4 \cdot 2)\end{array}$ \\
\hline $\begin{array}{ll}\text { Hypoxia: } & \\
1^{\circ} \mathrm{PHT} & (\mathrm{n}=5) \\
\text { Normal } & (\mathrm{n}=6)\end{array}$ & $\begin{array}{ll}67 \cdot 2 & (9 \cdot 6)^{\star \star} \\
25 \cdot 0 & (3 \cdot 8)^{\star \star \star}\end{array}$ & $\begin{array}{l}30 \cdot 2(4 \cdot 8)^{\star} \\
10 \cdot 0(1 \cdot 4)\end{array}$ & $\begin{array}{l}114 \cdot 6(6 \cdot 4) \\
102 \cdot 8(11 \cdot 0)^{\star}\end{array}$ & $\begin{array}{l}58 \cdot 8(4 \cdot 5) \\
56 \cdot 8(9 \cdot 2)^{\star}\end{array}$ & $\begin{aligned} 96 \cdot 6 & (8 \cdot 3)^{\star \star} \\
105 \cdot 3 & (3 \cdot 5)\end{aligned}$ \\
\hline $\begin{array}{cc}\text { Smoking: } & \\
1^{\circ} \mathrm{PHT} & (\mathrm{n}=3) \\
\text { Normal } & (\mathrm{n}=6)\end{array}$ & $\begin{array}{l}48 \cdot 3(14 \cdot 3) \\
15 \cdot 8(1 \cdot 6)\end{array}$ & $\begin{array}{r}21.7(7.6) \\
6.4(0.8)\end{array}$ & $\begin{array}{ll}136 \cdot 0 & (3 \cdot 5)^{\star \star} \\
125 \cdot 8 & (6 \cdot 7)^{\star \star}\end{array}$ & $\begin{array}{l}71.3(3.9) \\
78.4(5.6)\end{array}$ & $\begin{array}{c}99 \cdot 7(14 \cdot 3) \\
105 \cdot 0(2 \cdot 8)^{\star \star \star}\end{array}$ \\
\hline
\end{tabular}

$1^{\circ} \mathrm{PHT}$, data from patients with primary pulmonary hypertension; Normal, healthy volunteers.

Significance of change from resting values before interventions: ${ }^{\star} p<0.05,{ }^{\star} p<0.01,{ }^{\star}{ }^{\star \star} p<0.001$.

At all times pulmonary systolic and diastolic pressures were, as expected, significantly greater in $1^{\circ} \mathrm{PHT}$ patients than in healthy

volunteers $(\mathrm{p}=<0.005-\mathrm{p}<0.001)$. Systemic diastolic pressures differed between groups for "eating" only ( $p<0.001)$.

Systemic systolic pressures did not differ significantly between groups at any time.

Values given are for peak exercise, peak hypoxia (taken at $80 \%$ duration of intervention), 8 minutes of eating, and 5 minutes of smoking and are averaged over a mean time of 100 minutes for "resting".

both groups $(p<0.001$ for change from baseline for all pressures). Successive corresponding one minute mean values of pulmonary and systemic pressures during exercise were closely correlated in all patients (range of $r$ values $0.88-0.97, p<0.001$ for all). Plasma catecholamine concentrations rose normally with exercise (table 3 ) and peak values for noradrenaline $(6.27(0.24) \mathrm{nmol} / \mathrm{l}$ and adrenaline $(889.65(218.32) \mathrm{pmol} / \mathrm{l})$ did not differ significantly from those achieved in our healthy volunteers $(6.65(1.03) \mathrm{nmol} / \mathrm{l}$ and 622.21 (169.20) $\mathrm{pmol} / \mathrm{l}$ respectively).

\section{HYPOXIA}

Patients tolerated exposure to $10 \%$ oxygen in nitrogen for 4-20 minutes compared with healthy volunteers who tolerated $10 \%$ oxygen for 8-30 minutes. Systemic pressure did not alter significantly from resting values (fig 3, table 2). Arterial oxygen saturation fell significantly $(95.0(1.4)$ to $74.1(5.5) \% ; \mathrm{p}<0.001)$

Figure 2 Changes in pulmonary and systemic arterial pressures and heart rate (mean

(SEM)) with exercise in five patients with pulmonary hypertension. Standard errors were calculated for integrated mean pressures and heart rate from successive one minute intervals before, during, and after exercise. Exercise time varied between patients (shown as $0-100 \%$ on horizontal axis) and standard errors were calculated in $10 \%$ steps for duration of exercise (shown as continuous lines above mean systolic and below mean diastolic pressures, and a dotted line above mean heart rate).
Systemic arterial Systemic arterial
pressure $(\mathrm{mmHg})$

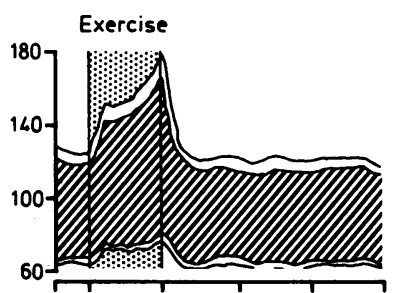

Pulmonary arteria
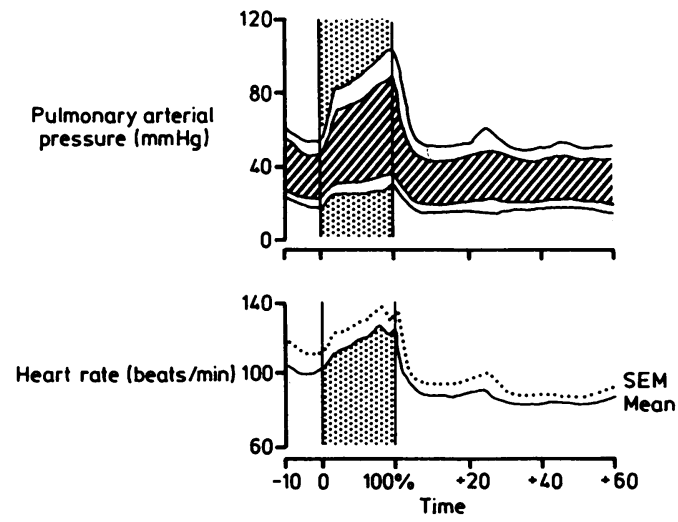
pressure ( $\mathrm{mmHg}$ )

by the end of the hypoxic period. Pulmonary pressure rose in all patients $(p<0.01$ and $p<$ 0.05 for systolic and diastolic values respectively, fig 3). Heart rate rose significantly (fig 3, table 2). Absolute increases in pulmonary pressure in the patients were significantly greater than in healthy volunteers $(p<0.001$ and $p<$ 0.01 for systolic and diastolic values respectively, table 2). However, time-group interaction analysis indicated parallel behaviour of pulmonary arterial pressure in both groups. Plasma noradrenaline was unchanged while adrenaline values rose significantly (table 3 ), similar to the response seen in healthy volunteers.
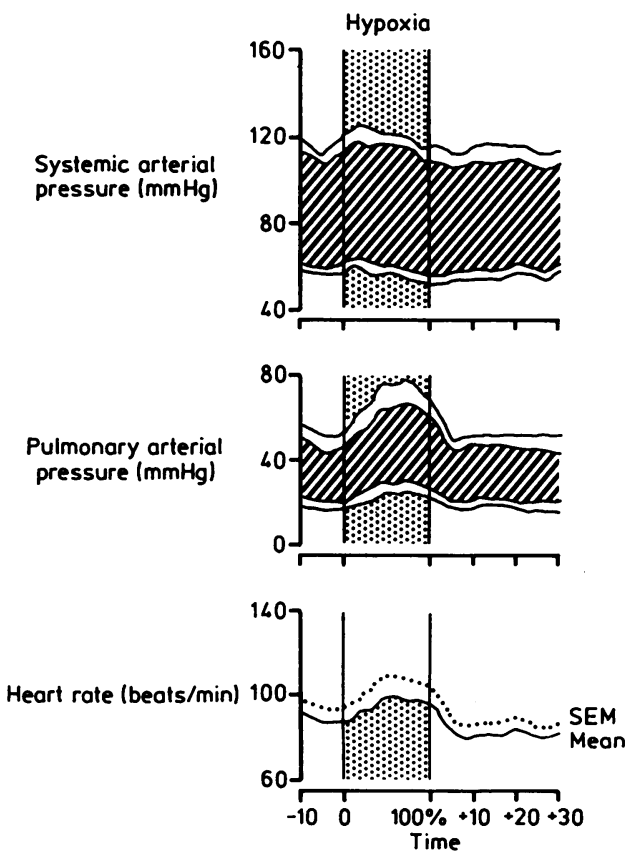

Figure 3 Changes in pulmonary and systemic arterial pressures and heart rate (mean (SEM)) with acute hypoxia in five patients with pulmonary hypertension. See fig 2 for symbols and calculation of plotted values. Duration of hypoxia varied between patients and is shown as $0-100 \%$ on the horizontal axis. 
Figure 4 Changes in pulmonary and systemic arterial pressures and heart rate (mean

(SEM)) when two cigarettes were smoked, between 0-10 min, by three patients with pulmonary hypertension. See fig 2 for variables, symbols, and calculation of plotted values.
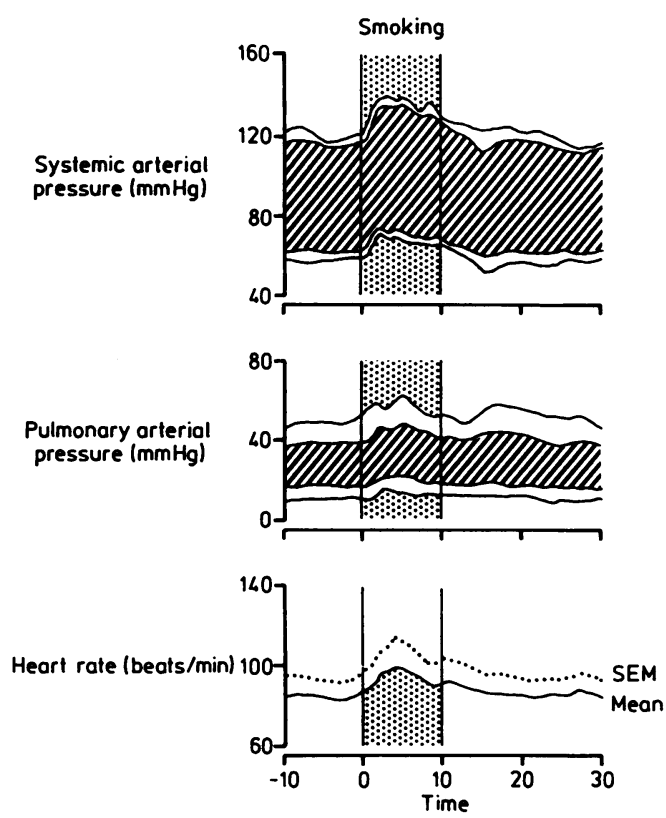

SMOKING

Three patients (smokers) smoked two standard cigarettes in 10 minutes. During smoking systemic pressure rose significantly above resting values and returned to baseline within five minutes of the patients finishing the cigarettes (fig 4). The tendency for pulmonary pressure and heart rate to increase was not statistically significant. These variables showed no discernible response in normal subjects. Patients retained a strong, positive correlation between pulmonary and systemic pressures throughout smoking (range of $r$ values, $r=0.69-0.88$, $\mathrm{p}<0.01$ to $\mathrm{p}<0.001)$. In all three patients both noradrenaline and adrenaline concentrations rose (table 3 ).

EATING

The tendency for the systemic and pulmonary pressures and heart rate to increase during eating did not achieve statistical significance (table 2). The overall pattern of haemodynamic responses was similar to that seen in healthy volunteers (table 2). Time-group interaction analysis showed no difference between the two groups.

\section{MISCELLANEOUS}

Quiet walking was associated with modest increases and stair-climbing with sharp increases in pulmonary pressure above resting values. Two patients slept for brief periods while sitting and all pressures and heart rates were notably lower at these times. An incidental observation was the lack of any cardiac arrhythmia beyond occasional supraventricular and ventricular extrasystolic beats during recordings.

\section{Discussion}

Knowledge of the fluctuations in pulmonary arterial pressure in health and disease is critical for interpretation of data obtained during catheterisation of patients with primary pulmonary hypertension. We used techniques enabling the computerised integration of continuous, beat to beat, direct recording of both pulmonary and systemic arterial pressure to acquire such data in patients with primary pulmonary hypertension during rest, free ambulation, and various interventions. Carefully standardised conditions of study allowed us to compare our findings with those in a group of healthy volunteers studied earlier. ${ }^{5}$

Over periods of 8-10 hours and in the course of several interventions and activities pulmonary pressure was maintained above normal values in these patients (fig 1, table 2). Absolute variation in pulmonary pressure was greater in primary pulmonary hypertension than in the healthy volunteers (table 1 ). Relative variation (coefficients of variation) were similar or less than in the volunteers (table 1) and somewhat greater than the $8 \%$ figure calculated by Rich et al for mean pulmonary pressure from intermittent readings in resting, supine patients. ${ }^{3}$ These data show the increased absolute, but not proportional, variability of pulmonary pressure in primary pulmonary hypertension. We have also confirmed earlier reports of sig-

Table 3 Plasma catecholamines (mean (SEM))

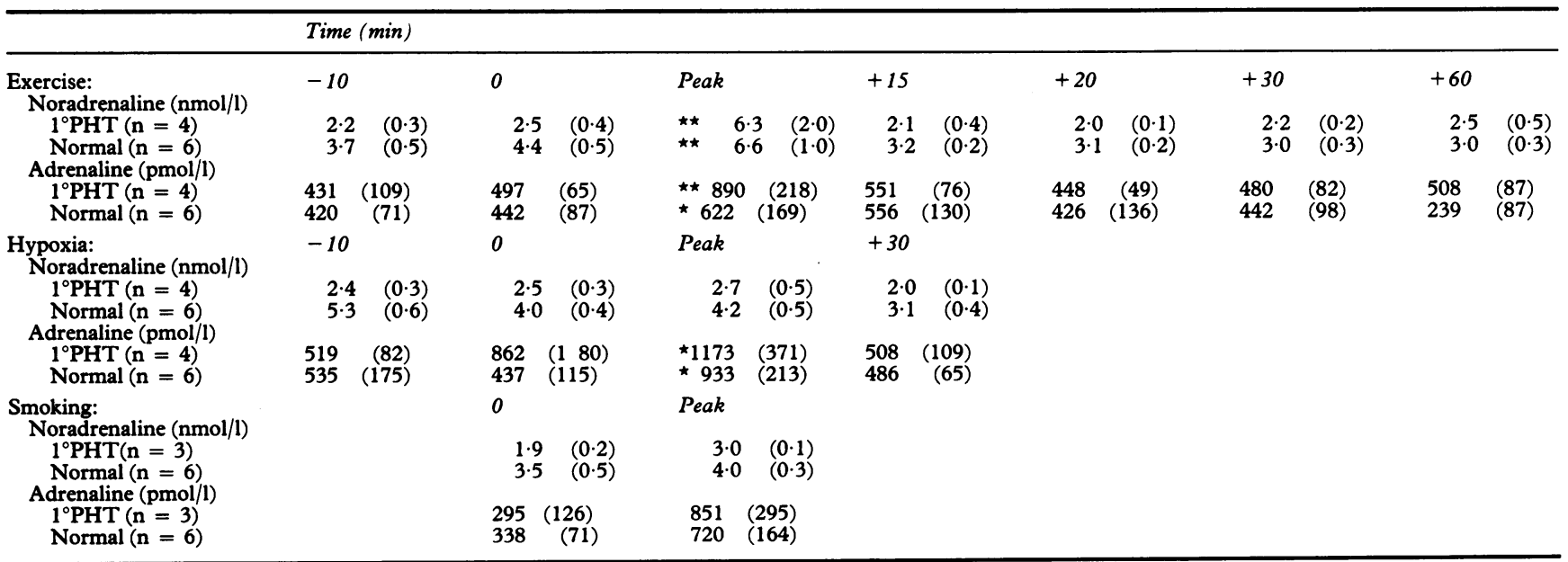

Within groups two way analysis of variance of all time points followed by paired $t$ test comparing 0 and peak values indicated a significant rise in both catecholamines with exercise, and in adrenaline alone during hypoxia for both groups.

${ }^{\star} \mathrm{p}<0.05,{ }^{\star \star} \mathrm{p}<0.01, \star \star \star \mathrm{p}<0.001$ 
nificant spontaneous fluctuations in pulmonary pressure in primary pulmonary hypertension apparently unrelated to medication or any other easily discerned stimulus. ${ }^{23}$

To our knowledge, variability in systemic pressure and the relation of systemic to pulmonary pressures have not previously been reported in primary pulmonary hypertension. Our data indicate that the values of variability in systemic pressure in our patients with primary pulmonary hypertension resembled those seen in healthy volunteers. These data imply that the lesion resulting in the high values of (and excessive absolute variation in) pulmonary pressures seen in this patient group exerts no similar effect on fluctuations in systemic pressure.

Changes in pulmonary pressures during exercise were directionally similar but of greater absolute magnitude than those seen in healthy volunteers. Hypoxia also resulted in a greater rise in pulmonary pressure than was seen in healthy volunteers. ${ }^{512}$ Over the whole study period we found generally parallel movement of pulmonary and systemic pressures in three patients, but no relation in two patients. In contrast, pulmonary and systemic pressures changed in parallel in all healthy volunteers. Although no clinical characteristics predicted the absence of this relation in the two patients, it may be related to the abnormal pulmonary vasculature.

The activity of the sympathetic nervous system has not previously been assessed in primary pulmonary hypertension. We continuously monitored heart rate and took frequent samples to estimate plasma concentrations of catecholamine at rest and during interventions. Despite the higher mean age of patients their baseline concentrations of plasma catecholamines tended to be lower than in the healthy volunteers and the peak values attained with interventions were similar in the volunteers and those with pulmonary hypertension. The pattern of change in plasma catecholamines with interventions was similar in both groups. Care must be taken in extrapolating from plasma catecholamine concentrations to sympathetic nervous activity and little can be divined concerning sympathetic traffic to any single organ..$^{13}$ Nevertheless, our data suggest that primary pulmonary hypertension is not attended by any gross alteration in either basal or stimulated global sympathetic nervous system activity.

Resting heart rates were similar in the two groups (table 2). However, it is noteworthy that during exercise, patients with primary pulmonary hypertension achieved slightly greater systemic pressures, and far greater pulmonary pressures than normal in association with substantially lower heart rates (table 2). Variability in heart rate also tended to be lower in the patient group (table 1). The groups were not well matched for age and the apparent diminished responsiveness in heart rate may simply reflect the greater age of the patients. An alternative explanation is that vagal inhibition of heart rate was enhanced as a result of increased stimulation of baroceptors in the pulmonary arteries or right ventricle ${ }^{14}$ in primary pulmonary hypertension. Further studies with age and weight matched controls, together with assessment of the effect of atropine, may clarify this issue.

In summary, our data indicate that the absolute variability of pulmonary arterial pressure and the magnitude of absolute changes in pulmonary arterial pressure in response to stimuli are enhanced in primary pulmonary hypertension. The variability of systemic arterial pressure is normal. Short term congruency of shifts in pulmonary and systemic pressures are preserved but in some patients the relation between systemic and pulmonary pressures, over periods of hours, is weakened. Basal and stimulated plasma catecholamine values are normal, suggesting preservation of normal sympathetic nervous system activity in primary pulmonary hypertension.

This study was supported by the National Heart Foundation and the Medical Research Council of New Zealand. We are grateful for the help of Special Tests, Biochemistry and Die Department Staff at The Princess Margaret Hospital and that of Hormone Assay Staff at Christchurch Hospital. Chris Frampton, Biostatistician, gave statistical advice. Mrs P Hollis gave secretarial assistance. AMR and IGC are senior fellows of the National Heart Foundation of New Zealand.

1 Hatano S, Straser T, eds. Primary pulmonary hypertension Report on a WHO meeting, October 1973. Geneva: World Health Organization, 1975 .

2 Rich S, Martinez J, Lam W, Rosen KM. Captopril as treatment for patients with pulmonary hypertension. treatment for patients with pulmonary hypertension.
Problem of variability in assessing chronic drug treatment. Br Heart J 1982;48:272-7.

3 Rich S, D'Alonzo GE, Dantzker DR, Levy PS. Magnitude and implications of spontaneous hemodynamic variability in primary pulmonary hypertension. Am J Cardiol 1985; 55:159-63.

4 Ikram H, Richards AM, Hamilton EJ, Nicholls MG. Continuous recording of pulmonary artery pressure in unrestricted subjects. Br Heart J 1984;51:421-6.

5 Richards AM, Ikram H, Nicholls MG, Espiner EA. Hamilton EJ, Richards RD. Ambulatory pulmonary arteria pressures in humans: relationship to arterial pressure and hormones. Am J Physiol 1986;25:H101-8.

6 Rich S, Dantzker DR, Ayres SM, et al. Primary pulmonary hypertension. A national prospective study. Ann Intern Med 1987;107:216-23.

7 Millar-Craig MW, Hawes D, Whittington JR. New system for recording ambulatory blood pressure in man. Med Bio Eng Comput 1978;16:727-31.

8 Patterson JA, Naughton J, Pietras RJ, Gunnar RM. Treadmill exercise in assessment of the functional capacity of patients with cardiac disease. Am J Cardiol 1972;30: patients

9 Peuler JD, Johnson GA. Simultaneous single isotope radioenzymatic assays of plasma norepinephrine, epinephrine and dopamine. Life Sci 1977;6:625-33.

10 Mancia G, Ferrari A, Gregorini L, et al. Blood pressure and heart rate variabilities in normotensive and hypertensive human beings. Circ Res 1983;53:96-104.

11 Snedecor GW, Cochran WG. Statistical methods. 6th ed. Ames, Iowa: Iowa State University Press, 1967.

12 Westcott RN, Powler NO, Scott RC, Hauenstein VD McGuire J. Anoxia and human pulmonary vascula resistance. J Clin Invest 1951;30:957-70.

13 Esler MD. Assessment of sympathetic nervous function in humans from noradrenaline plasma kinetics. Clin Sci 1982;62:247.

14 Miller MR, Kasahara M. Studies on the nerve endings in the heart. Am J Anat 1964;115:217-34. 